\title{
A comparison of age difference reaction to computer interface
}

\author{
Chadi Riman • Husam Ghusn • Eric Monacelli
}

Received: 14 October 2009 /Accepted: 4 August 2010/Published online: 12 August 2010

(C) European Group for Research into Elderly and Physical Activity (EGREPA) 2010

\begin{abstract}
In this work, a simplified interface system for education of people with disabilities is presented. This platform allows the control of different tools (PC games, robot, and electric wheelchair) using different interfaces (mouse, joysticks, etc.). This interface system can be automatically reconfigured to match user's ability, and it is operated locally or remotely through the internet. Saving of experimental progress data for later analysis can be done. Quantitative indicators are defined for evaluation purposes and to determine the needed assistance and adaptation. The system is tested on different age groups for people without physical disability. Results are presented and discussed.
\end{abstract}

Keywords Elderly · Training · Computer interface

\section{Introduction}

Computer education for people with special needs is a challenge for our modern society which is more dependable on computer technologies. Using a computer sounds like a trivial task for most of us but is a difficult task for someone

\footnotetext{
C. Riman $(\bowtie)$

Fahad Bin Sultan University,

Tabuk, Saudi Arabia

e-mail: chadi_riman@yahoo.com

H. Ghusn

AinWazein Hospital,

AinWazein, Lebanon

e-mail: husam.ghusn@awh.org.lb

E. Monacelli

University of Versailles,

Versailles, France

e-mail: eric.monacelli@lisv.uvsq.fr
}

who cannot properly control his hand muscles. This applies on people with certain types of motor disabilities (such as motor cerebral deficiency) and also on elderly people who start to lose flexibility in handling their hands muscles. Ageing is accompanied with the loss of the muscle flexibility and thus a computer interface may not be as easy to use for an elderly person as it seems to us.

The "Platform for Interface and Assistance Evaluation" (PLEIA as French title acronym) was created to evaluate and assist people with special need on the usage of a computer interface. PLEIA was developed for many types of disabilities, including people with cerebral palsy or motor cerebral deficiency, post-stroke patients, quadriplegic patients, and persons who cannot control their movements because of weakness or spasm (myopathy, athestosy). Patients use PLEIA via various kinds of input devices such as a mouse, a haptic joystick with force feedback, a trackball, a head tracker, or others. This allows them to control computer-simulated systems (PC games), real objects (robots and toys), and other mobility aids (electric wheel chair or walker). PLEIA can also be used by the occupational therapist and the medical team. For this purpose, quantitative indicators were defined and used for evaluation, for determining the needed assistance and adaptation, and for comparing user's performance. The software facilitates the saving of experimental data (time, position, distance to target, and collisions) in a database for analysis and sharing. It can be automatically reconfigured to match the user's ability, and is operated locally or remotely through internet or intranet connections [1, 2]. PLEIA can also be used to generate configurations and/or test scenarios for different devices.

In this work, a special attention was given to the elderly people. Thus, we worked on applying PLEIA on different healthy age groups to test the difference in terms of 
performance. "Existing systems" section introduces PLEIA, "PLEIA description" section defines its quantitative indicators, "Quantitative evaluation indicators" section describes the experiment, and, finally, the experiment results are shown in "Application examples" section.

\section{Existing systems}

Nowadays, there are many available systems whose purpose is to treat and help people with disabilities. Most of These systems, however, suffer from certain limitations.

The system presented in [3] evaluates the "point and click" ability of a user based on many criteria and provides assistance to reach a target through a haptic interface. Two other low-cost telerehabilitation systems were introduced in [4]: the first uses a force feedback joystick with simple twodimensional (2D) games, whereas the second uses a force feedback driving wheel with three-dimensional (3D) driving exercises. Another telerehabilitation platform [5] is intended for patients with a stroke-induced disability. This platform uses several haptic devices (joysticks, driving wheels, pointing devices, keyboard) and includes remote (Internet) accessibility and videoconferencing. In [6], a telerehabilitation system for arm and hand therapy following stroke is presented. It consists of a Web-based library of status tests, therapy games, and progress charts, and can be used with a variety of input devices, including force feedback joysticks. In [7], Technical and Patient Performance Using a Virtual Reality-Integrated Telerehabilitation System is described. The system consists of the Rutgers Ankle prototype robot, a local PC and a remote PC over the Internet. In [8], exercises were built and used on a laptop with a force feedback joystick and a steering wheel for measuring motor dysfunction in Parkinson's disease. The exercises consist of tracking a continuously moving target (pursuit tracking), or moving to a predetermined target (step tracking). A haptic interface for hand evaluation and rehabilitation was developed in [9]. This system uses the DataGlove Rutgers Master II (RMII). Another system called "Rutgers Ankle" was also developed for lower extremity rehabilitation [9]. The built-in software provides exercises for different cases: Rubber ball exercise for strengthening the hand of a patient, Virtual RMII exercise for the rehabilitation of post-stroke patients, and Virtual Airplane exercise for lower extremity rehabilitation. Virtual reality by means of a head-mounted display was used in [10] for the purpose of improving patient walking skills through training with computer-generated obstacles. In [11], a low-cost telerehabilitation system for upper limb dysfunction was presented. An example of a virtual driving environment was also shown using a commercial force feedback driving wheel. In [12], an exercise system combining arm ergometry with video gaming, called the
GAME/sup Cycle, was developed. A haptic device with two active degrees of freedom and a tendon-driven transmission system was developed in [13].

PLEIA, as compared to other systems in the same area, has the following integrated features:

1. PLEIA integrates a set of tools into a single platform, while most other systems are focused on one or few tools or tasks.

2. PLEIA has an assistance mode which helps the patient in reaching a goal. This feature was only found in [3].

3. PLEIA has an adaptation mode which automatically reconfigures the system according to the patient's ability.

4. PLEIA has a supervision service which allows the therapist to control and follow up a session locally or remotely. A limited version of this feature was found in [7].

\section{PLEIA description}

PLEIA was introduced in a previous work $[14,15]$. PLEIA is a platform which has two main purposes: Evaluate a person's dexterity to use a computer-pointing device and manipulate a mobility aid such as an electric wheel chair. Different tests are used to achieve these goals. Patients' tests can be applied using different interfaces such as joystick (with or without force feedback), mouse, trackball, and head tracker. These interfaces are used to control 2D computer-simulated exercises, 3D objects (robots, toys), and mobility aids (electric wheel chair, walker). The software facilitates the saving of a patient's exercise test data in a database for analysis and sharing. It can be automatically reconfigured to match the user's ability, and can also be used to generate configurations and/or test scenarios for different devices [14, 15]. PLEIA is added into the middle of the standard therapy session (Fig. 1) which consists of the user, the therapist, the system (such as computer or wheel chair) and the peripheral device (such as joystick, mouse, or other control devices).

PLEIA can be accessed locally or remotely through internet or intranet connections [1,2]. Many users can be simultaneously connected to the server where the experiment is being conducted. It is, therefore, possible to remotely

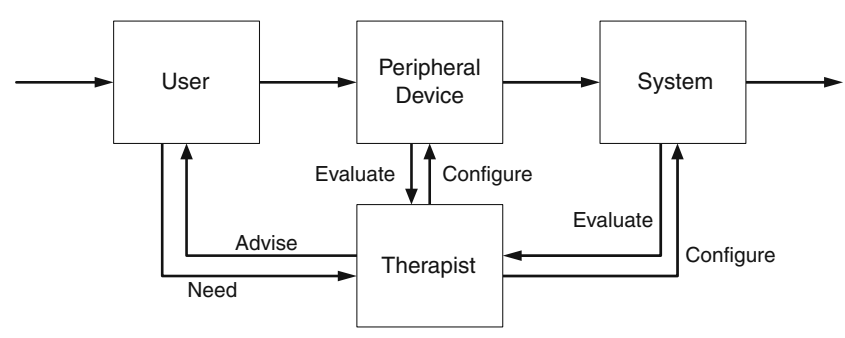

Fig. 1 Strategy for a normal therapy session 


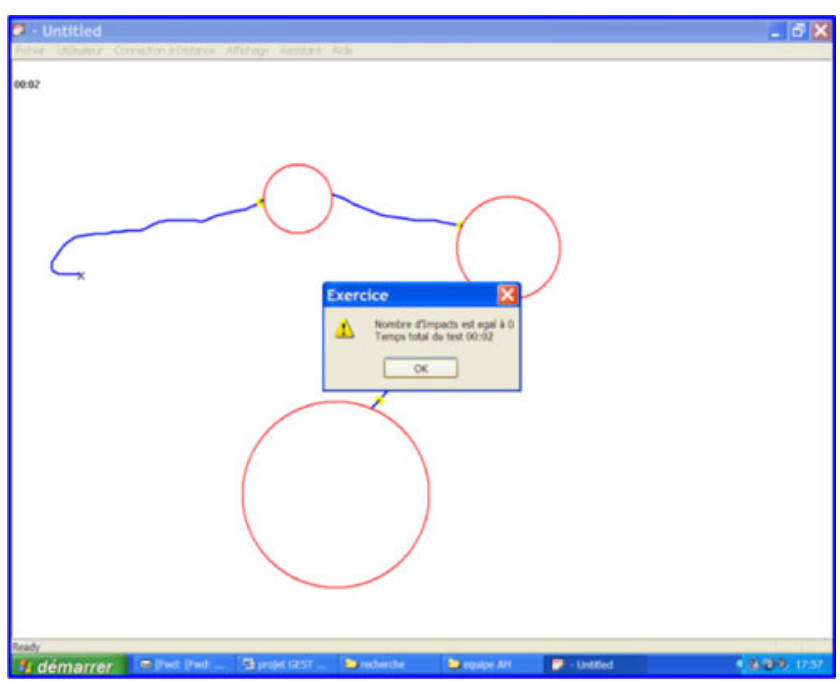

Fig. 2 PLEIA exercise: reach/click targets

execute a test, modify experiment parameters, chat between server and clients, exchange scenarios and experiment parameters, and help the user in achieving a goal by using another pointing device (joystick, mouse, or others) in parallel.

PLEIA can also be used by the occupational therapist or medical team. For this purpose, quantitative indicators are defined ("PLEIA description" section) and used for evaluation, for determining the needed assistance and adaptation, and for comparing user's performance. The upcoming subsections describe the PLEIA functions and the creation of test scenarios.

\section{PLEIA functions}

PLEIA supports the control of two-dimensional computersimulated exercises. Four types of exercises are proposed

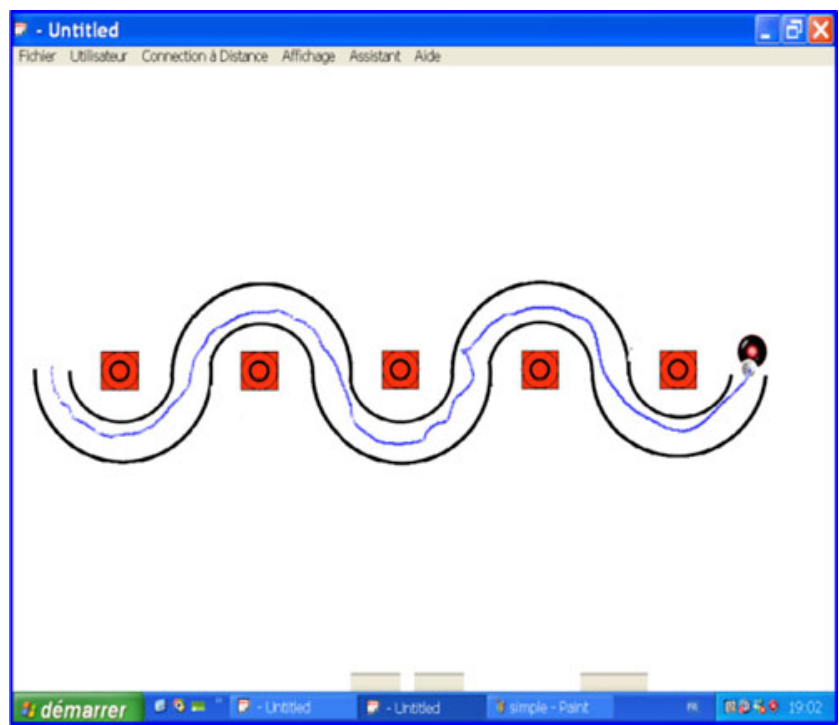

Fig. 3 PLEIA exercise: follow path

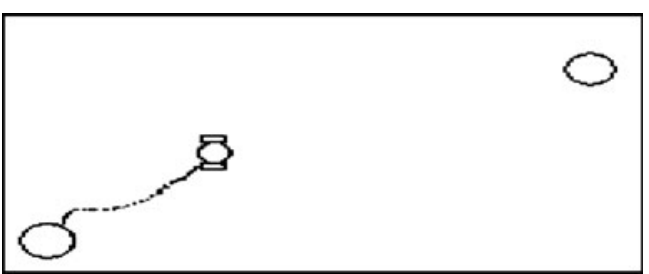

Fig. 4 PLEIA exercise: drag and drop

by an occupational therapist team: The "Reach Targets" exercise (Fig. 2) which requires reaching all targets in a specified order, showing the next target in green and the others in red. The "Click Targets" exercise (Fig. 2) is similar to "Reach Targets" exercise but which requires, in addition, clicking at each reached target. The "Follow Path" exercise (Fig. 3) requires reaching all the targets while following a drawn path. In the "Drag and Drop" exercise (Fig. 4), it is required to reach a target, pick it up, move it, and drop it in the next target. These computer exercises have two modes of operation: PLEIA abstract and PLEIA tuned. In PLEIA abstract mode, the user manipulates abstract objects (circles and rectangles) on the computer screen, which is appropriate for most types of evaluations. In PLEIA tuned mode, the user can select images that fill screen objects, or choose a special game background and a moving cursor shape (Fig. 5). This mode is preferred by children according to therapist's recommendations.

PLEIA offers four different services: evaluation service, supervision service, assistance service, and the adaptation service. The evaluation service is used to provide an online or offline evaluation of a user. The data used for evaluation is collected online and analyzed. The frequency of data collection is $10 \mathrm{~Hz}$. An online feedback about an exercise performance can also be sent to the user in visual form, audio form, or through vibrations.

The supervision service is used by the therapist in order to supervise a session. The supervision can be online during the test or offline by examining and analyzing test results after the test. It can be local on the same computer and location, or remote at another computer or location. PLEIA can handle two input devices at the same time (such as two joysticks or one joystick and one mouse), on the same computer, or on two networked computers. This feature allows the patient and the supervisor to cooperate in achieving a certain goal. For example, the patient can get

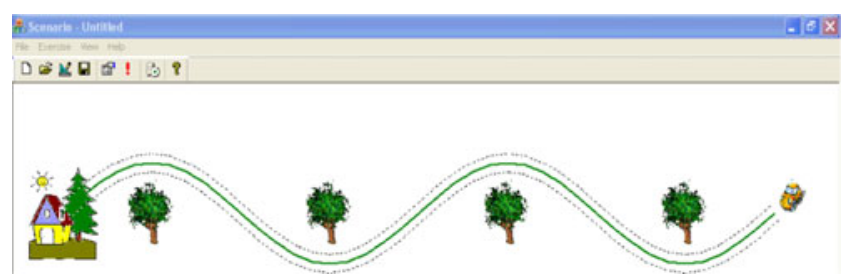

Fig. 5 PLEIA tuned 


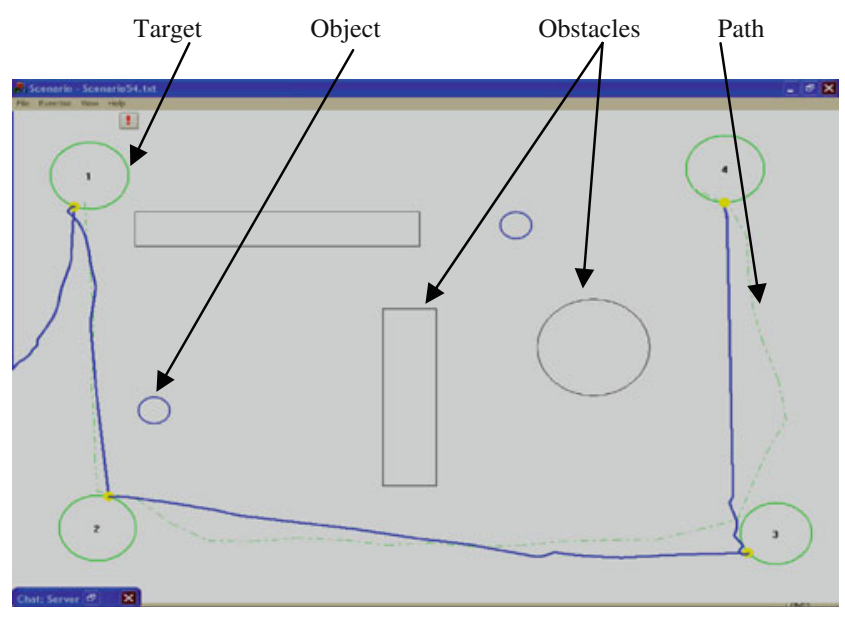

Fig. 6 PLEIA multi-platform software

force assistance from the supervisor joystick (human user help) to reach the targets. It is also possible to have a remote chat between patient and supervisor.

The assistance service is used to provide assistance or training to the user during an exercise in order to reach a goal. This type of assistance is computer-generated assistance which is different from the above mentioned humansupervised assistance. For example, the user can get force assistance from the joystick to reach a target. This force can be an attraction force toward a target or a path, or a repulsion force to avoid an obstacle. Finally, the adaptation service is used to compensate a user's deficiency such as an inability to move the joystick in one direction. This mode uses a fuzzy FIS controller. Assistance and adaptation can only be achieved when using a device with force feedback (joystick).

\section{Creation of test scenarios}

A test scenario consists of different components used in a test (Fig. 6) such as targets, objects, obstacles, and paths. Test scenarios can be generated, saved in text files for future use, and copied to other computers. A test scenario can also be modified by a therapist. The therapist can also change the test configuration such as the speed, the
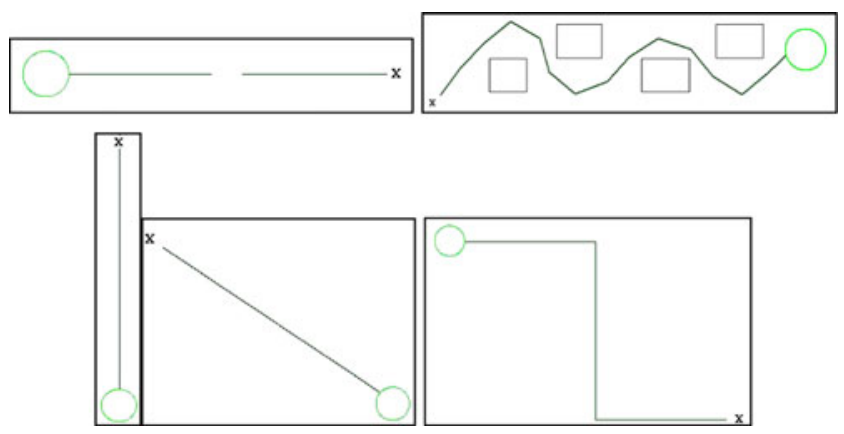

Fig. 75 simple tests applied at AinWazein Hospital

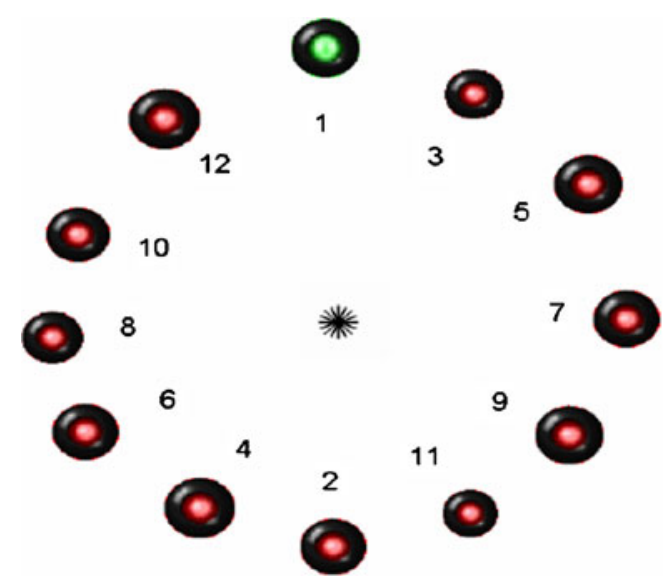

Fig. 8 Patient suggested test by therapist

feedback distance from obstacles, the sound effects, the pointing interface device, and others. Such changes are important for proper therapy sessions.

\section{Quantitative evaluation indicators}

An important feature of PLEIA is the quantitative indicators used for analyzing test results. The advantage of these quantitative indicators is to reduce the error of subjective assessments. It is therefore possible to quantitatively evaluate a patient's performance, compare two patients' performances, and record the evolution of a patient's performance.

The time indicator measures the total test accomplishment time. The resting time factor measures the percentage of inactivity during the test with respect to total test time. The distance efficiency indicator measures the minimum possible distance divided by the total actual test distance. The collisions indicator measures the total number of collisions with different obstacles during a test if it includes obstacles.

Other indicators are defined in PLEIA. We called them comfort indicators. Comfort indicators evaluate the user ability in manipulating the input device (joystick, mouse, trackball, etc.). Given the test trajectory $\left(x\left(t_{i}\right), y\left(t_{i}\right)\right)$, two

Fig. 9 Path test scenario applied in AinWazein hospital

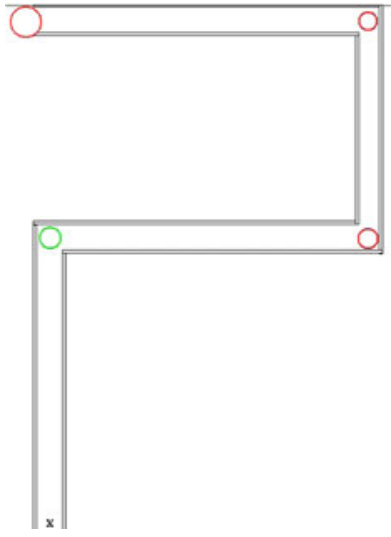


Fig. 10 Twelve targets test for Jamal (25 years): a first try, b last try

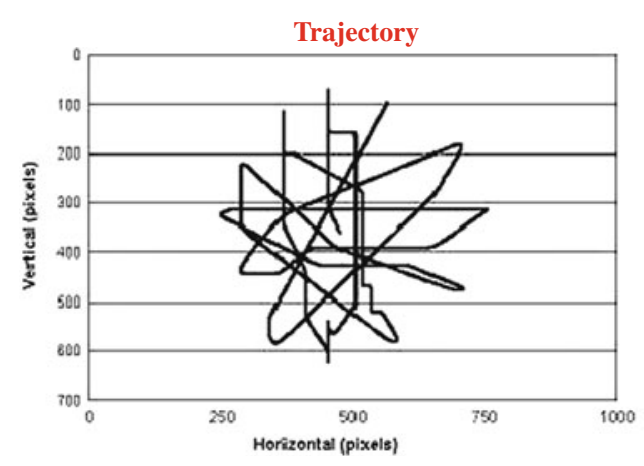

comfort indicators are defined: charge of command and regularity of command. The charge of command indicator measures the variation of the direction of the effort applied by the user on the input device during a test. A small variation indicates little effort exercised by the user. The regularity of command indicator measures the movement uniformity during a test. Whenever the movement's speed is nearly constant, the command is more regular. If the speed varies with time or the user stops often during a test, the command loses some regularity.

\section{Application examples}

Our investigation was set in a Lebanese elderly care center and hospital. Our work started in an elderly care center at AinWazein Hospital (Chouf, Mount Lebanon). Our work was supervised by a geriatrics doctor. His research questions included the following:

- Numbers of tries before reaching maximum performance

- Difference of performance among different age groups

- Changes in performance with time

- Relation between mental status and performance

- How can PLEIA help learning the use of an electric wheel chair?

The present study was done in order to answer the second question. Other application examples will be performed in the future to answer other questions. The test was performed on different age groups of people. The group was set on 10 years division: $20-29,30-39,40-49,50-59,60-69,70$ 79 , and $80-89$. Subjects from the current hospital employees and visitors were asked for participation. They were all healthy subjects with no motor disability. The total number of subjects is 50 because we could not find any participant in the 80 s group, and a part of the upper age groups was not filled. The gender distribution was $56 \%$ male and $44 \%$ female. The goal was to compare the reaction to computer pointing device (mainly a joystick interface) with different age groups from young to old people. Two PLEIA game scenarios were used for this purpose. One which is a standard manipulation and navigation experiment used by different therapists [3], and the other is passing the computer game pointer through a simple path similar to an existing path in the hospital. The tests were done on ten healthy people without disability from each age group. They were compared and presented in the next section.

Five simple test scenarios were used to make users learn how to use PLEIA (Fig. 7). Then two main tests were performed. The first test is a standard test designed by a therapist to reach 12 targets in a specific order without obstacles (Fig. 8) [3]. The second test is a simulation to a path in the hospital which connects the elderly center to the outpatient department (Fig. 9). The main goal is to pass the computer pointer through this path with minimum number of impacts against the path's wall.
Fig. 11 Path test for Jamal (25 years): a first try, b last try
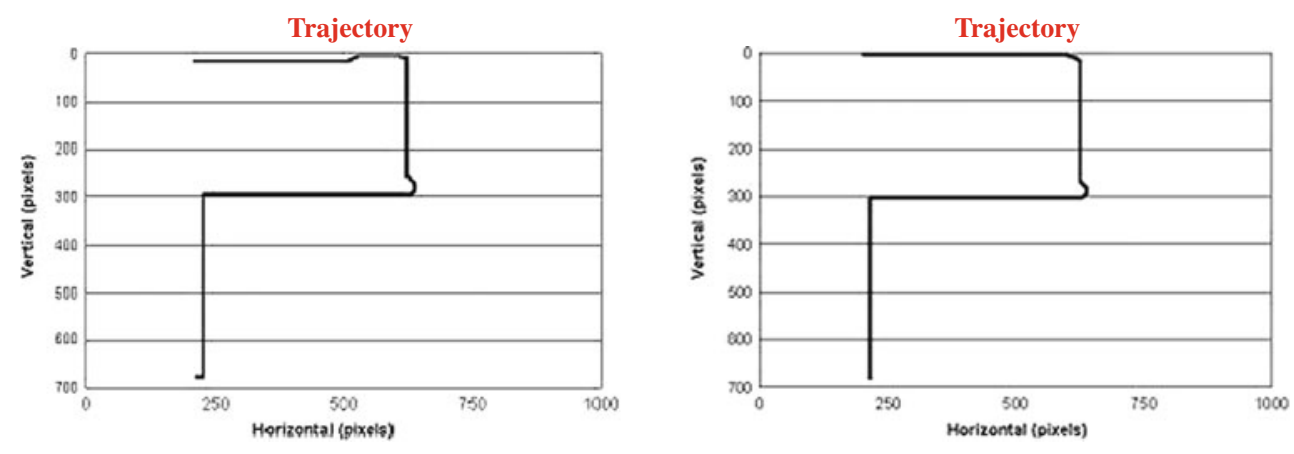
Fig. 12 Twelve targets test for Farid (75 years): a first try, b last try

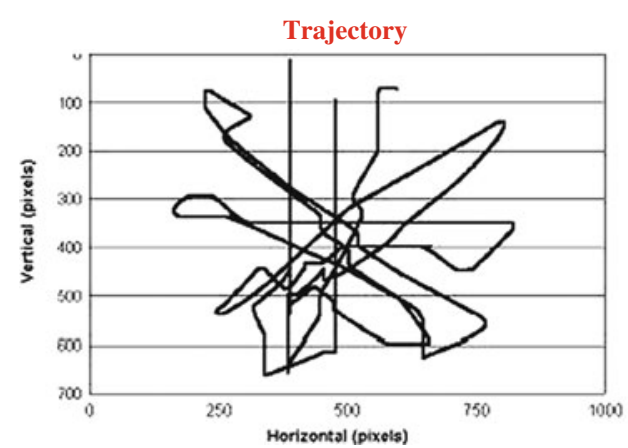

PLEIA 2D computer exercise was used with Force Feedback Joystick interface. The experiment procedure was the following:

- Explain the general idea to the user

- Explain the procedure to the user and show him what to do

- Perform the test once in front of the user

- Let the user try the experiment with our help twice without saving the results.

- Start the test and save the results. Help was only given orally to the user.

- Repeat each test five times for each user. The average results were taken into account in order to compensate for any learning curve or transient mistakes that might occur.

\section{Experiment results}

We managed to perform the tests for six age groups: 20-29, 30-39, 40-49, 50-59, 60-69, and 70-79 years. Fifty-eight persons participated in doing these tests but only 50 actually did all the tests: ten subjects from each group, except 60s (only six) and 70s (only four). We will first compare the performance between one person from age group 20-29 and another from age group 70-79. Next, we will present the average and standard deviation of all age groups outcomes.
Comparison of two individuals from two different age groups

Jamal is a young female of 25 years old. She did all the tests without any problem. Her learning phase was very short. All her tries were good. In the first test scenario with 12 targets, her total time went down from 78 to $76 \mathrm{~s}$. The trajectory was good for both tries (Fig. 10).

In the path scenario, her total time went down from 39 to $29 \mathrm{~s}$. The trajectory was good for both tries. The big time difference was due to her fear to hit the path walls during the first time. All her movements were regular (Fig. 11).

Farid is a 75-year-old man. He had some problems in performing the tests. Although the learning phase was short and the majority of his tries were good, he could not repeat the test more than twice. He stopped in the middle of the third try due to fatigue. In the first test scenario with 12 targets, his total time went down from 151 to $84 \mathrm{~s}$. The trajectory was better in the second try. The reason for the time difference is due to the fact that he did many oscillations and stops in the first try (Fig. 12).

In the path scenario, his total time went down from 38 to $32 \mathrm{~s}$. He did many oscillations in the first try, but he managed to do well on his last try. His movements were regular (Fig. 13).

\section{Comparison of the average of all age groups}

All data resulting from the experiments were analyzed using the mean and standard deviation tests. It was discovered that
Fig. 13 Path test for Farid (75 years): a first try, $\mathbf{b}$ last try
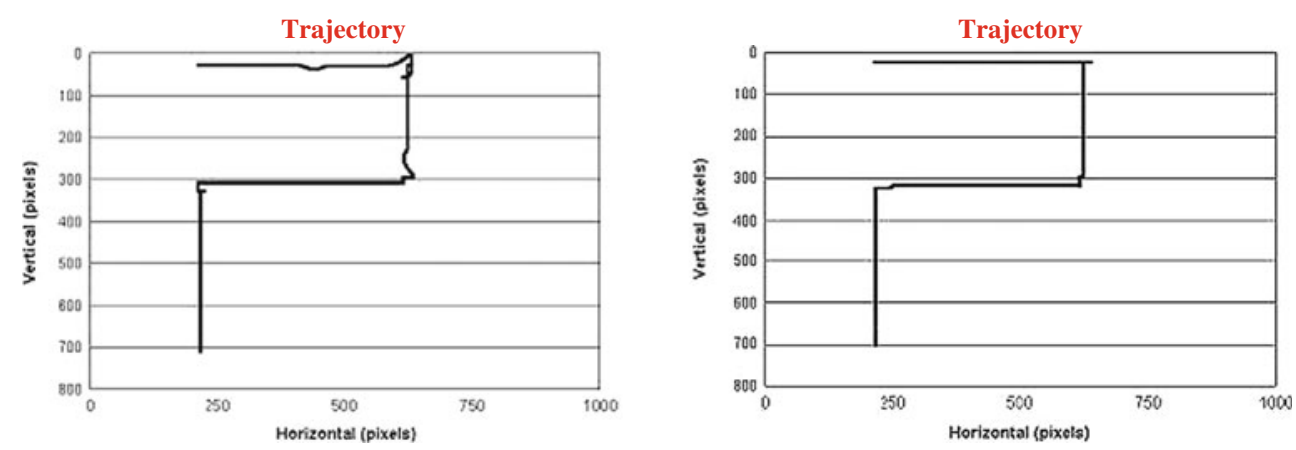


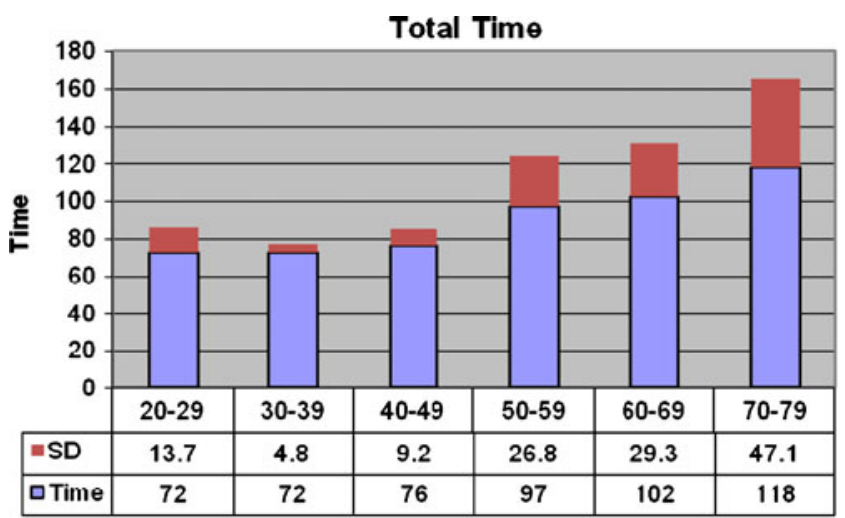

(a) Age

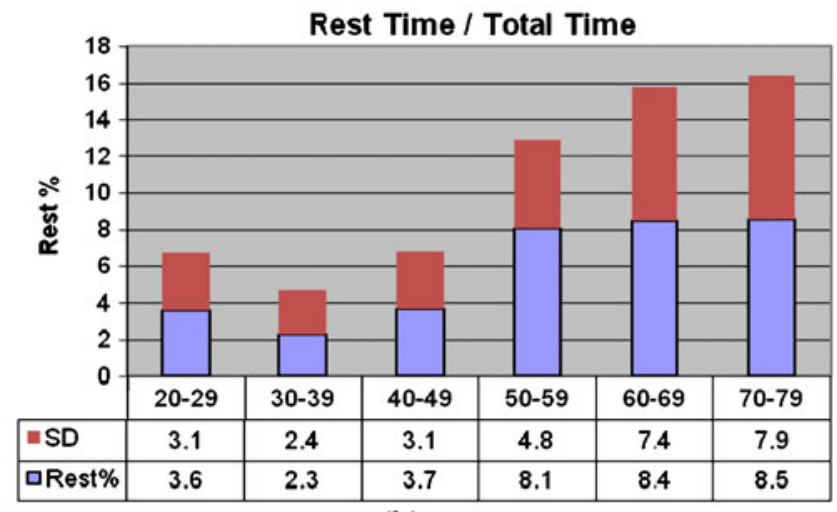

(b) Age

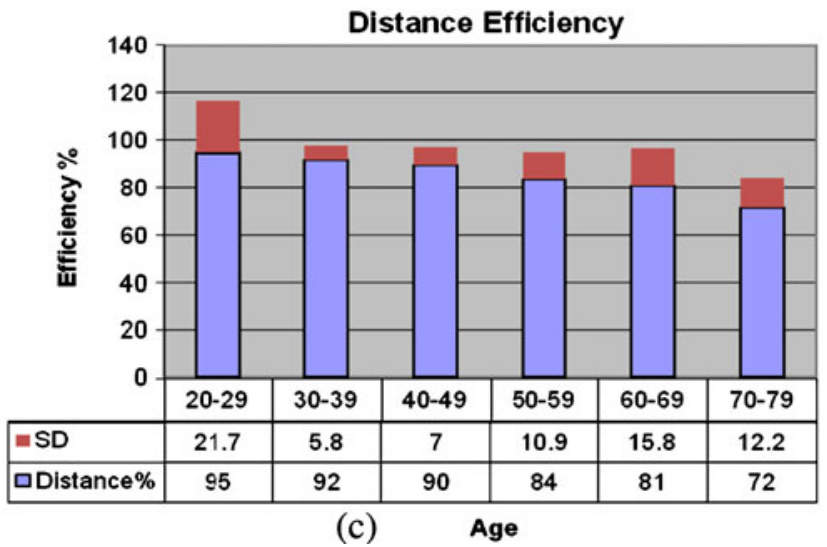

Fig. 14 Twelve targets test results: a total time, b resting time factor, c distance efficiency (having $N=10$ for all groups except 60 s (six) and 70 s (four))

data samples are coherent since the standard deviation is relatively small. After analyzing the average results taken for all subjects over the different age groups (Fig. 14), we discovered the following for the first experiment (12 targets):

- Total test time increased from an average of $72 \mathrm{~s}$ for the 20-29 age range to the average of $118 \mathrm{~s}$ for the $70-79$ age range.

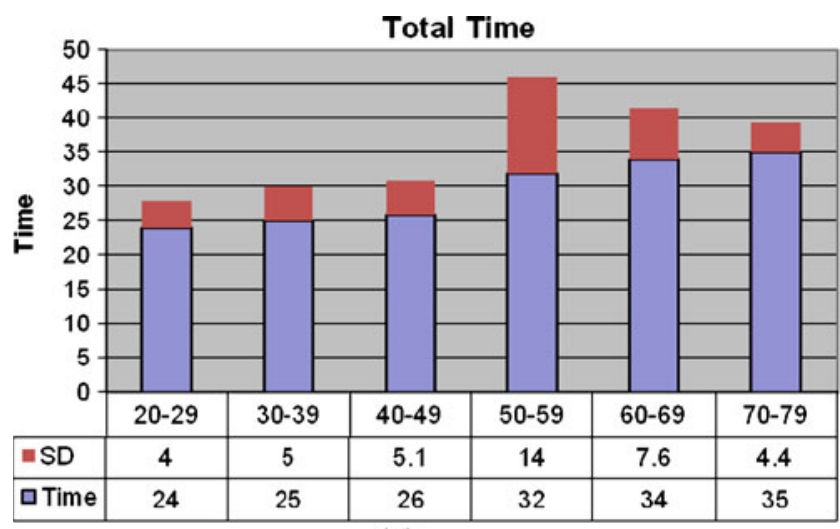

(a) Age

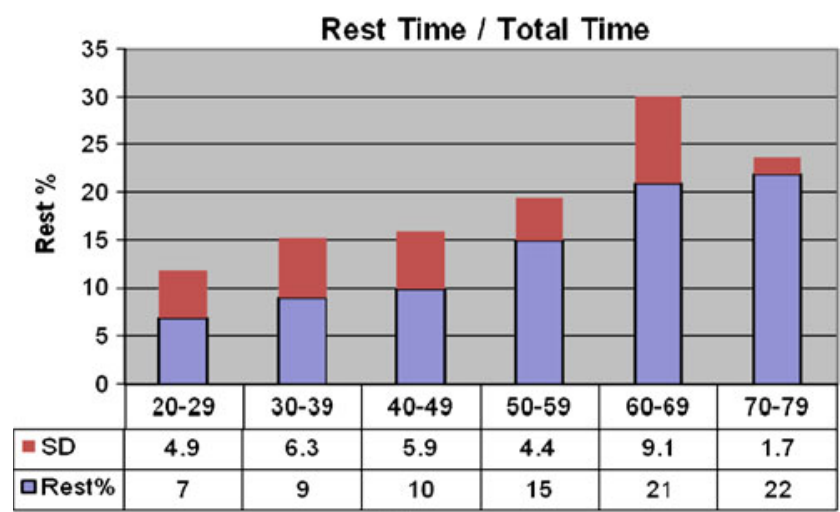

(b) Age

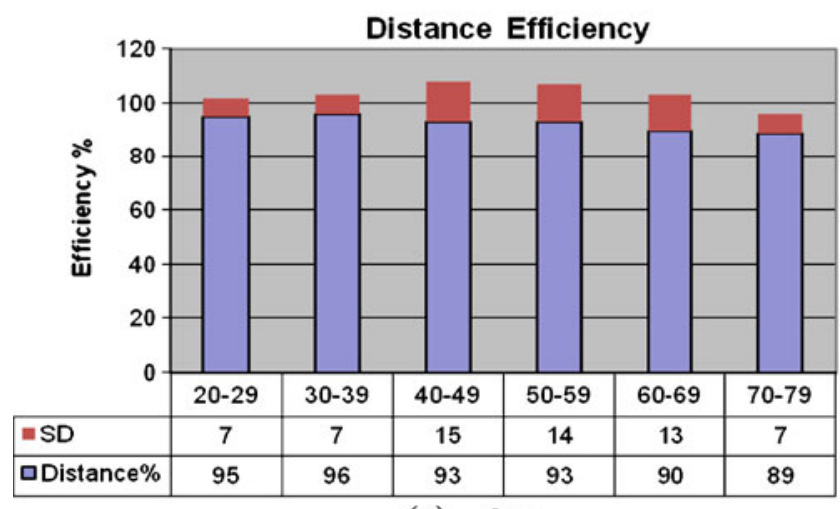

(c) Age

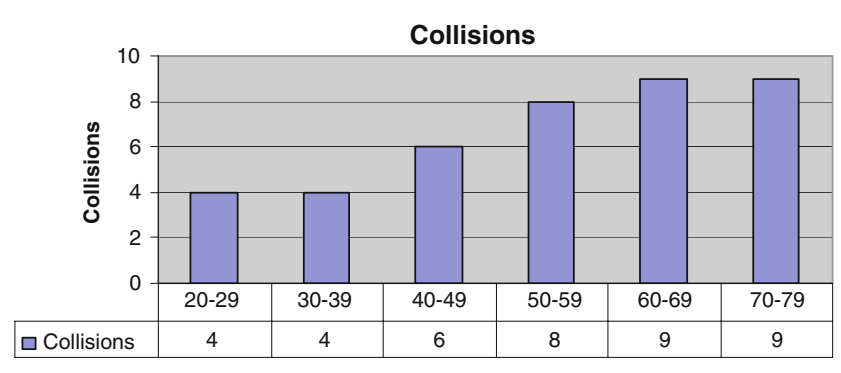

(d) Age

Fig. 15 Pathway test results: a total time, b resting time factor, c distance efficiency, $\mathbf{d}$ collisions $(N=10$ for all groups except $60 \mathrm{~s}$ and 70s) 
- The resting time factor increased from an average of $3.6 \%$ for the 20-29 age range to the average of $8.5 \%$ for the 70-79 age range.

- The distance efficiency decreased from an average of $95 \%$ for the $20-29$ age range to the average of $72 \%$ for the 70-79 age range.

- The collisions indicator cannot be measured because no obstacles are available in this experiment.

For the second experiment (Fig. 15):

- Total test time increased from an average of $23 \mathrm{~s}$ for the 20-29 age range to the average of $35 \mathrm{~s}$ for the $70-79$ age range.

- The resting time factor increased from an average of 7\% for the 20-29 age range to the average of $22 \%$ for the $70-79$ age range.

- The distance efficiency decreased from an average of $95 \%$ for the $20-29$ age range to the average of $89 \%$ for the 70-79 age range. We remark that the distance did not significantly change due to the fact that the path is very tight and the user does not have much freedom to deviate from minimum path.

- The number of collisions increased from an average of four for the 20-29 age range to the average of nine for the 70-79 age range.

The geriatrics doctor analyzed these results using chisquare statistical method to deduce the significant groups and suggested the following:

- The most significant age groups can be shown as two: the young 20-39 (merging the first two groups) and old 6079 (last two groups). Other groups can be disregarded.

- The above two age partitions have different results with different indicators.

- The total time indicator clearly shows that the young category spend less time to achieve the goal than the old category. The older people may be moving slower or stopping more often to think their way.

- The rest time indicator shows that the older people are stopping often. This can due to tiresome or to slower reflexes when taking decisions and changing paths.

- The distance efficiency shows no big difference. This difference, however, is due to the older people having less dexterity using their hand muscles in order to change directions.

- The results are very promising and further indicators may be suggested to improve the analysis such as:

- Rest time before first move

- Rest time after first move

- Number of rests

- Action time/quantity/amplitude

- Movement continuity

\section{Conclusion}

In this work, PLEIA evaluation and assistance multiplatform for people with disabilities is presented. PLEIA is currently operational and under evaluation by designers, therapists, and patients.

PLEIA was used to compare different age groups of healthy people without physical disability. The obvious outcome showed that elderly people have less control of their hand and arm muscles than young people. A more important result would be to adapt the used interface in order to better suit an elderly person (using PLEIA adaptation service, for example). Other devices are being tested. Ongoing studies on other devices (mouse, trackball) are being done and compared with joystick device to determine the most suitable device for each user.

Furthermore, the current results can be used as a base for future experiments that will include different types of motor disabilities. The results for people with disabilities can therefore be compared to our current results and give better assessment of the person's physical situation.

\section{References}

1. Mougharbel I, El Hajj A, Artail H, Riman C (2006) Remote lab experiments models: a comparative study. Int J Eng Educ 22 (4):849-857

2. El Hajj A, Mougharbel I, Riman C (2004) On the design and implementation of remote computer controlled laboratory experiments. CIBITIC Conference, Beirut, Lebanon

3. Keates S, Hwang F et al. (2002) Cursor measures for motionimpaired computer users. Proceedings of the Fifth International ACM Conference on Assistive Technologies 2002, Edinburgh, Scotland

4. Burdea (2004). Invited paper: low-cost telerehabilitation. Proceedings of Third Int. Workshop on Virtual Rehabilitation, Switzerland, pp 3297-3300

5. Feng X, Winters J (2005) Progress towards a service-oriented universal access telerehabilitation platform. Proceedings of the 2005 IEEE 9th International Conference on Rehabilitation Robotics, Chicago, IL, USA, pp 357-360

6. Reinkensmeyer DJ, Pang CT, Nessler JA, Painter CC (2002) Webbased telerehabilitation for the upper extremity after stroke. IEEE Trans Neural Syst Rehabil Eng 10(2):102-108

7. Deutsch JE, Lewis JA, Burdea G (2007) Technical and patient performance using a virtual reality-integrated telerehabilitation system: preliminary finding. IEEE Trans Neural Syst Rehabil Eng 15(1):30-35

8. Allen DP, Playfer JR et al (2007) On the use of low-cost computer peripherals for the assessment of motor dysfunction in parkinson's disease-quantification of Bradykinesia using target tracking tasks. IEEE Trans Neural Syst Rehabil Eng 15 (2):286-294

9. Burdea G (2001) Haptic feedback interfaces for rehabilitation. Proceedings State of the Science Conference on Telerehabilitation and Applications of Virtual Reality, Washington DC, pp $51-56$

10. Jaffe D (2001) Using virtual reality and telerehabilitation to improve walking in individuals following stroke. Proceedings 
State of the Science Conference on Telerehabilitation and Applications of Virtual Reality, Washington DC, pp. 57-59

11. Jadhav C, Krovi V (2004) A low-cost framework for individualized interactive telerehabilitation. Proceedings of the 26th Annual International Conference of the IEEE EMBS, San Francisco, CA, USA, pp 3297-3300

12. Songfend G, Grindle GG et al (2006) Development and qualitative assessment of the GAME/sup Cycle/ exercise system. IEEE Trans Neural Syst Rehabil Eng 14(1):83-90
13. Mali U, Goljar N, Munih M (2006) Application of haptic interface for finger exercise. IEEE Trans Neural Syst Rehabil Eng 14 (3):352-360

14. Peralta $\mathrm{H}$, Riman $\mathrm{C}$ et al. (2006) A reconfigurable evaluation and assistance platform for handicapped. IROS 2006. Beijing, China, pp 247-252

15. Peralta H, Riman C et al. (2007) A reconfigurable evaluation, rehabilitation, learning and assistance platform for handicapped people: PLEIA, Ed. RAS. Rehabilitation Robotics, ISBN, 2007G 